\title{
Demo Abstract: Heterogeneous Social Sensing Edge Computing System for Deep Learning based Disaster Response
}

\author{
Daniel (Yue) Zhang, Dong Wang \\ Department of Computer Science and Engineering \\ University of Notre Dame, Indiana, USA \\ yzhang40@nd.edu,dwang5@nd.edu
}

\begin{abstract}
Social sensing has emerged as a new application paradigm where measurements about the physical world are collected from humans or devices on their behalf. One of the representative application of social sensing is disaster damage assessment (DDA) that automatically identifies damage severity of impacted areas from imagery reports reported by eyewitness in the aftermath of a disaster (e.g., earthquake, hurricane, landslides). In this demo, we present a Social Sensing based Edge Computing system (SSEC) that can coordinate the privately owned IoT devices in close proximity of the disaster scene to collect, process and report the real-time status of the disaster. We showcase a supply chain-based resource management framework for SSEC that tames the pronounced run-time and hardware heterogeneity of the IoT devices at the edge to provide reliable sensing and computing power. The system is demonstrated on a real-world hardware platform consists of a diverse set of heterogeneous embedded systems.
\end{abstract}

\section{CCS CONCEPTS}

- Human-centered computing $\rightarrow$ Collaborative and social computing; • Computing methodologies $\rightarrow$ Distributed computing methodologies; $\bullet$ Computer systems organization $\rightarrow$ Embedded and cyber-physical systems;

\section{KEYWORDS}

edge computing, social sensing, disaster response, disaster damage assessment, resource management

\section{INTRODUCTION}

Social sensing is an emerging application paradigm where humans and devices on their behalf report observations about the events in the physical world $[3,5]$. In this paper, we focus on an important social sensing application - deep learning-based disaster damage assessment (DDA) where deep neural network approaches are used to automatically identify damage severity of impacted areas from social media images in the aftermath of a disaster (e.g., earthquake, hurricane, landslides) $[1,4]$. The assessments are then delegated to emergency response agencies (e.g., FEMA and police departments)

Permission to make digital or hard copies of part or all of this work for personal or classroom use is granted without fee provided that copies are not made or distributed for profit or commercial advantage and that copies bear this notice and the full citation on the first page. Copyrights for third-party components of this work must be honored

For all other uses, contact the owner/author(s).

IoTDI '19, April 15-18, 2019, Montreal, QC, Canada

(c) 2019 Copyright held by the owner/author(s).

ACM ISBN 978-1-4503-6283-2/19/04.

https://doi.org/10.1145/3302505.3312589 to adopt appropriate countermeasures. Traditionally, the DDA applications are performed in a centralized fashion - the raw imagery data collected from the IoT devices is directly sent to a back-end server for processing. This back-end based solution often causes excessive bandwidth consumption and unnecessary delay $[7,10]$.

In this demo, we focus on a Social Sensing based Edge Computing (SSEC) paradigm for DDA where the IoT devices owned by individuals are used as the computational resource at the edge to accomplish the disaster damage assessment tasks. The SSEC paradigm has several unique advantages. First, SSEC provides a more economical and scalable edge computing solution by leveraging the devices owned by end users. Second, the SSEC devices form a mobile sensor network to accomplish the sensing tasks that are challenging for infrastructure/static sensors [9]. Third, SSEC is more suitable for the delay-sensitive DDA application by pushing the computation and service to the edge where the data reside. Finally, SSEC reduces the risk of overloading the back-end servers and avoids the single point of failure in the system [2].

Despite its benefits, SSEC also faces a critical challenge in DDA applications: heterogeneity. This challenge is motivated by the observation that the edge devices in SSEC are owned by individuals and often have diversified computational power, run-time environments, network interfaces, and hardware equipment. Such heterogeneity poses significant challenges in the resource management of SSEC systems in DDA applications. Examples of such challenges include masking the pronounced heterogeneity across diverse platforms, allocating interdependent DDA tasks with complex resource requirements, and adapting to the dynamic and diversified execution contexts of the edge devices.

In this demo, we showcase a new resource management framework, HeteroEdge [8], which addresses the heterogeneity of SSEC by 1 ) providing a uniform interface to abstract the device details (hardware, operating system, CPU) owned by individuals; and 2) effectively allocating DDA tasks to heterogeneous edge devices.

\section{THE DEMO}

\subsection{System Setup}

The demonstration prototype of the SSEC system consists an edge server and 4 edge devices. The edge server is a personal laptop with Quad core i5-7300 processor and 8 GB of RAM. The edge server is used to coordinate the communication and task allocations of the edge devices and also provide additional storage and processing power at the edge. The edge devices include 1 Nvidia Jetson TX2 board, 1 Jetson TX1 board, and 2 Raspberry Pi3s. A setup of the experiment platform is shown in Figure 1. These edge devices are representative of systems commonly used in portable computers, 
smart sensors, UAVs, and autonomous cars. The edge devices and the servers are interconnected via a wireless router.

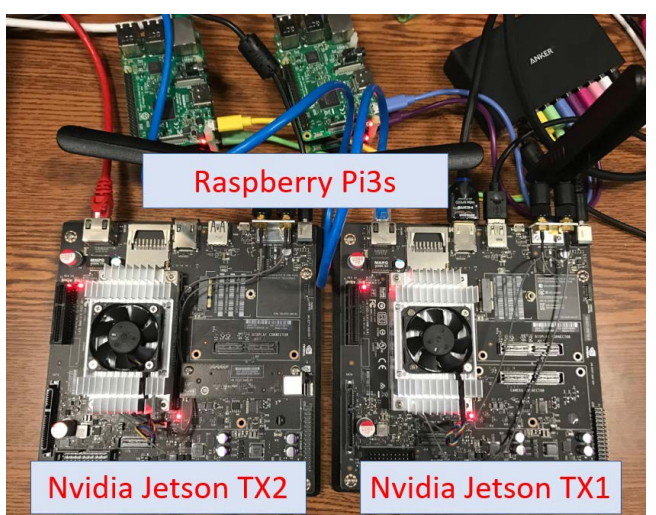

Figure 1: SSEC Demo Platform

One key performance evaluation metric is the energy consumption of the edge devices. We use an INA219 Current Sensor IC, interfaced to an Arduino Uno Micro-controller board.

\subsection{Application and Dataset}

We demonstrate our heterogeneous edge computing system through a real-world social sensing based DDA application. The DDA application consists of the following tasks: i) capturing the live images from locations of interest by leveraging the edge devices equipped with cameras; ii) extracting Damage Detection Map (DDM) features using Convolutional Neural Network (CNN) model from raw images; iii) assessing the damage severity from DDM using the algorithm in [1]. The goal of the HeteroEdge is to assign these tasks to a collection of heterogeneous edge devices that minimizes the energy consumption of the edge devices and the end-end delay for the application [8]. In the demoed application, edge devices are tasked to sense and evaluate the levels of damages at different locations in the aftermath of a disaster by analyzing the image data collected from social media. We will use 2,000 images related to the Ecuador Earthquake in 2016 from Twitter as the dataset.

\subsection{The Demonstration}

The demonstration will mainly focus on comparing and contrasting different task allocation strategies in supporting the delay sensitive social sensing applications described above. We choose three representative resource management schemes in SSEC. The first one is Bottom-Up Game Theoretic Task Allocation (BGTA) which is the state-of-the-art resource management scheme that lets edge devices to pick tasks using a game-theoretical model [6]. The second one is random assignment (RAND) where each DDA task is randomly assigned to one of the edge devices. The third scheme is HeteroEdge which leverages a supply-chain based model to identify the optimal task offloading path from a set of heterogeneous devices for the interdependent DDA tasks.

The demo will provide the functional validation of HeteroEdge from two perspectives. First, we will demonstrate the system performance by showing the deadline hit rate (DHR) and the classification effectiveness (in terms of Accuracy, Precision, Recall, and F-1 Score) of the DDA application. This will validate that HeteroEdge can effectively meet the QoS requirement of the DDA application. Second, we will evaluate the energy consumption of edge devices. The energy-aware design of HeteroEdge allows the edge devices to pick the tasks that yield the minimum delay while maximizing energy savings. The demo will report the average energy consumption of each edge device. An interactive front-end will be developed using Python for visualizing and interacting with the SSEC system. The interface will display the metrics designed above and the current status (CPU frequency, memory usage, power consumption) of each edge device.

\section{CONCLUSION}

In conclusion, this demo showcases an heterogeneous edge computing system for disaster damage assessment application on a real-world SSEC testbed. We will perform the functional validation of HeteroEdge, a novel resource management system we recently developed. We will demonstrate the effectiveness of HeteroEdge in achieving both QoS and energy savings.

\section{ACKNOWLEDGMENT}

This research is supported in part by the National Science Foundation under Grant No. CNS-1831669, CBET-1637251, CNS-1566465 and IIS-1447795, Army Research Office under Grant W911NF-171-0409, Google 2017 Faculty Research Award. The views and conclusions contained in this document are those of the authors and should not be interpreted as representing the official policies, either expressed or implied, of the Army Research Office or the U.S. Government. The U.S. Government is authorized to reproduce and distribute reprints for Government purposes notwithstanding any copyright notation here on.

\section{REFERENCES}

[1] Xukun Li, Huaiyu Zhang, Doina Caragea, and Muhammad Imran. 2018. Localizing and Quantifying Damage in Social Media Images. arXiv preprint arXiv:1806.07378 (2018)

[2] Weisong Shi, Jie Cao, Quan Zhang, Youhuizi Li, and Lanyu Xu. 2016. Edge computing: Vision and challenges. IEEE Internet of Things fournal 3, 5 (2016), 637-646.

[3] Dong Wang, Tarek Abdelzaher, and Lance Kaplan. 2015. Social sensing: building reliable systems on unreliable data. Morgan Kaufmann.

[4] Dong Wang, Tarek Abdelzaher, Lance Kaplan, and Charu C Aggarwal. 2013. Recursive fact-finding: A streaming approach to truth estimation in crowdsourcing applications. In Distributed Computing Systems (ICDCS), 2013 IEEE 33rd International Conference on. IEEE, 530-539.

[5] Dong Wang, Boleslaw K Szymanski, Tarek Abdelzaher, Heng Ji, and Lance Kaplan. 2018. The age of social sensing. Accpeted in IEEE Computer (2018).

[6] Daniel Zhang, Yue Ma, Yang Zhang, Suwen Lin, X Sharon Hu, and Dong Wang. 2018. A real-time and non-cooperative task allocation framework for social sensing applications in edge computing systems. In 2018 IEEE Real-Time and Embedded Technology and Applications Symposium (RTAS). IEEE, 316-326.

[7] Daniel Zhang, Yue Ma, Chao Zheng, Yang Zhang, X Sharon Hu, and Dong Wang. 2018. Cooperative-Competitive Task Allocation in Edge Computing for DelaySensitive Social Sensing. In 2018 IEEE/ACM Symposium on Edge Computing (SEC). IEEE, 243-259.

[8] Daniel Zhang, Tahmid Rashid, Xukun Li, Nathan Vance, and Dong Wang. 2019. HeteroEdge: Taming The Heterogeneity of Edge Computing System in Social Sensing. In Internet-of-Things Design and Implementation (IoTDI), 2019 ACM/IEEE Third International Conference on. ACM. accepted.

[9] Daniel Zhang and Dong Wang. 2019. An Integrated Top-down and Bottom-up Task Allocation Approach in Social Sensing based Edge Computing Systems. In IEEE INFOCOM 2019-IEEE Conference on Computer Communications. IEEE. accepted.

[10] Yang Zhang, Nathan Vance, Daniel Zhang, and Dong Wang. 2018. Optimizing Online Task Allocation for Multi-Attribute Social Sensing. In The 27th International Conference on Computer Communications and Networks (ICCCN 2018). IEEE. 